\title{
The limitations of small outcome studies
}

\author{
Raymond J. Gibbons, MD, and David Hodge, $\mathrm{MSc}^{\mathrm{b}}$
}

See related article, pp. 277-284

In this issue of the Journal, Koh et $\mathrm{al}^{1}$ report a single-center study in which they attempt to relate the findings on myocardial perfusion images (MPI) performed for preoperative evaluation prior to non-cardiac surgery to subsequent cardiac events. In an earlier study, ${ }^{2}$ the authors had reported that the most common source of inappropriate referrals to MPI in their institution was pre-operative risk stratification. However, they also found that $40 \%$ of these inappropriate studies were abnormal. They, therefore, designed this study to examine the relationship between MPI appropriateness, MPI findings, and subsequent clinical outcomes. Their novel and provocative hypothesis was that MPI findings would predict outcome in appropriate studies, but not in inappropriate studies.

Koh et al are to be commended for this novel study and their hypothesis. Their emphasis on clinical outcomes as the primary outcome in this study is laudatory. In the current era of quality improvement and performance measurement, clinical outcomes are increasingly predominant. Performance measures that focus on " "process" or "surrogates" are being de-emphasized. Likewise, in the ongoing discussion related to healthcare reform, a focus on value in healthcare now dominates. Value is often defined in terms of patient outcomes, satisfaction, and safety, adjusted for cost over a span of care. $^{3}$

Unfortunately, studies which focus on clinical outcomes require considerable larger sample sizes than clinical research which utilizes other endpoints. The power of any study to detect differences in outcome, or

From the Division of Cardiovascular Disease, Department of Internal Medicine, ${ }^{\mathrm{a}}$ Division of Biomedical Statistics and Informatics, Department of Health Services Research, ${ }^{\mathrm{b}}$ Mayo Clinic, Rochester, MN.

Reprint requests: Raymond J. Gibbons, MD, Division of Cardiovascular Disease, Department of Internal Medicine, Mayo Clinic, Gonda 5, 200 First Street S.W, Rochester, MN 55905; gibbons. raymond@mayo.edu.

J Nucl Cardiol 2012;19:230-2.

$1071-3581 / \$ 34.00$

Copyright $@ 2012$ American Society of Nuclear Cardiology.

doi:10.1007/s12350-012-9521-1 to define important determinants of outcome, is primarily determined not by the sample size, but rather by the number of events. Thus, the sample size required in a population with a low incidence of subsequent events, such as patients with mild symptoms and stable coronary artery disease, is considerably larger than the sample size required in a population with a high event rate, such as patients with advanced heart failure. Outcome studies are therefore considerably more difficult to perform, particularly on a single-center-basis, and generally require both larger sample sizes and longer follow-up than most investigators recognize.

Koh et al chose to focus on major cardiac and cerebrovascular events (MACCE) during 90 days of follow-up. Given the short follow-up, and generally low rate of cardiac or cerebrovascular events at the time of non-cardiac surgery in current practice, their study hypothesis demanded a very large sample size.

Unfortunately, the group studied by Koh et al was only 176 patients, and not all of these underwent noncardiac surgery. Although they do not provide the actual number of patients who proceeded to non-cardiac surgery, it can be estimated from Figure 2 as between 100 and 110. Not surprisingly, there were no events in the remaining patients who did not undergo non-cardiac surgery. The overall number of events reported by Koh et al was only $11-7$ non-fatal myocardial infarctions, 3 deaths, and 1 stroke.

Given this small number of events, and the general rule of thumb that one should identify a single significant independent variable for each 10 events, this study was seriously under-powered to test the hypothesis. A major determinant of the risk of cardiac events at the time of non-cardiac surgery is the type of surgery. ${ }^{4}$ Koh et al do not provide us with a rigorous multi-variable analysis of the determinants of MACCE or the number of patients in each surgical risk group. However, their overall results would seem to confirm the importance of the risk of surgery, as the 90-day MACCE event rates for high-risk surgery (64\%) far exceed the event rates which they report for intermediate-risk surgery patients with poor functional class (13\%), intermediate-risk surgery patients with good functional class $(4 \%)$, or lowrisk surgery patients $(0 \%)$. Given their small number of events, it would be unlikely that any other factor, such as normal/abnormal scan, or appropriateness, would be independently significant after consideration of surgical 
Table 1. Event rates by SSS with $95 \% \mathrm{CI}$

\begin{tabular}{llllll}
\hline & & $\mathbf{0}$ & $\mathbf{1 - 5}$ & $\mathbf{6 - 1 0}$ & $\mathbf{2 1 1}$ \\
\hline $\mathrm{N}$ & 176 & 111 & 16 & 19 & 30 \\
Events & 11 & 3 & 2 & 2 & 4 \\
Event rate (\%) & & 2.7 & 12.5 & 10.5 & 13.5 \\
Cl reported by Koh (\%) & & $1.6-5.3$ & $8.6-19.5$ & $8.3-11.2$ & $2.9-18.9$ \\
CI from SAS $(\%)$ & $0-5.7$ & $0-28.7$ & $0-24.3$ & $1.2-25.6$ \\
\hline
\end{tabular}

risk. In fact, identification of a second important variable with only 10 events would likely be spurious.

The data in outcome studies are often presented in terms of event rates. However, it must be recognized that the estimates of event rates will be subject to broad 95\% confidence limits when the sample size is small and there are few events. Thus, the inclusion of $95 \%$ confidence intervals (CIs) for event rates is critical for interpretation. To their credit, Koh et al attempted to provide these confidence limits in Table 2 and in Figures 4 and 6 . In their statistical methods, Koh et al indicate that they used the Statistical Package for Social Sciences (SPSS) system for data analysis. This reviewer is not familiar with SPSS, but would question the accuracy of the results presented by Koh et al. The more commonly employed SAS package ${ }^{5}$ provides estimates of the $95 \%$ CIs which are consistent with the CIs based on proportions that are shown in standard statistical texts. ${ }^{6}$ Shown in Table 1 is a comparison of the event rate and the 95\% CIs according to the summed stress score (SSS), as reported by Koh and estimated from SAS. The width of the CIs determined by the SAS package is broader than those reported by Koh et al. For example, for the 111 patients with a SSS of 0 , there were 3 events, for reported event rate of $2.7 \%$. Koh et al report a $95 \% \mathrm{CI}$ of 1.6 to 5.3. In contrast, the estimate provided in the SAS system for the $95 \%$ CI is 0 to 5.7. For the 16 patients with a low-risk SSS, there were 2 events, for an event rate of $12.5 \%$. Koh et al report a 95\% CI of 8.6 to 19.5. In contrast, the estimate provided by the SAS package for this CI is $0 \%$ to $28.7 \%$. From the SAS CIs, it can be readily appreciated that there is broad overlap between the CIs for different SSSs. Although this finding appears to contradict other larger studies in the literature, it is not a surprising result for the small sample size and the small number of events.

The data reported in Figure 4 have similar limitations. There were 34 low-risk surgical patients (with no MACCE) and 36 intermediate-risk surgical patients with good functional class (with one MACCE). We do not know how many of these patients actually proceeded to surgery. If all 34 low-risk surgical patients had proceeded to surgery, the upper $95 \%$ exact confidence limit for the $0 \%$ event rate would be $10.3 \%$. (The upper CI would be even larger if fewer patients went to surgery.) There appears to be an error in the reported event rate for the 36 intermediate-risk surgical patients with good functional class. Given the single event in that group, if all 36 patients went to surgery, the event rate should be $2.7 \%$ with $95 \%$ confidence limits of 0 to 8.2. If fewer patients went to surgery, the event rate, as well as the upper confidence limit, would be even higher.

As properly reported by Koh et al, their study has other limitations. They could not control the clinical decision to proceed with surgery, or the measurement of biomarkers and ECGs following surgery. This may certainly have led to ascertainment bias, if patients with abnormal scans or high-risk surgery were more likely to be tested post-operatively than patients with low-risk scans or surgery. They also could not control the management of patients during or after surgery. We do know how many of these patients received beta blocker therapy during the peri-operative period.

Given these limitations, and the wide confidence limits on the reported event rates, what can the evidence-based reader conclude from this study? First, outcome studies in relatively low-risk cohorts such as this one are very difficult. They require very large sample sizes to have sufficient cardiac events (and therefore statistical power) to answer important clinical questions. Second, the data presented here appear to support the long-standing observation that the inherent risk of surgery is a major determinant of cardiac events at the time of non-cardiac surgery. Finally, although the authors' hypothesis is meritorious, and certainly deserves further study in much larger groups, the data presented here do not provide a definitive answer to this important question. The low event rate that they report in patients with inappropriate tests may reflect a lack of statistical power rather than a truly low event rate.

\section{Conflict of interest}

Dr Gibbons is a consultant for Lantheus Medical Imaging. 


\section{References}

1. Koh AS, Flores JLS, Keng FYJ, Tan RS, Chua TSJ. Correlation between clinical outcomes and appropriateness grading for referral to myocardial perfusion imaging for preoperative evaluation prior to non-cardiac surgery. J Nucl Cardiol 2012. doi:10.1007/s12350011-9462-0.

2. Koh AS, Flores JL, Keng FY, Tan RS, Chua TS. Evaluation of the American College of Cardiology Foundation/American Society of Nuclear Cardiology appropriateness criteria for SPECT myocardial perfusion imaging in an Asian Tertiary cardiac center. J Nucl Cardiol 2011;18:324-30.

3. Smoldt RK, Cortese DA. Pay-for performance or pay for value? Mayo Clin Proc 2007;82:210-3.

4. Fleisher LA, Beckman JA, Brown KA. ACC/AHA 2007 guidelines on perioperative cardiovascular evaluation and care for noncardiac surgery: a report of the American College of Cardiology/American Heart Association Task Force on Practice Guideline (Writing Committee to Revise the 2002 Guidelines on Perioperative Cardiovascular Evaluation for Noncardiac Surgery): developed in collaboration with the American Society of Echocardiography, American Society of Nuclear Cardiology, Heart Rhythm Society, Society of Cardiovascular Anesthesiologists, Society for Cardiovascular Angiography and Interventions, Society for Vascular Medicine and Biology, and Society for Vascular Surgery. Circulation 2007;116:e418-99.

5. SAS Institute Inc., Cary, NC, Version 9.2.

6. Glantz SA. Primer of biostatistics. New York, NY: Mc-Graw Hill, Inc.; 1997. 